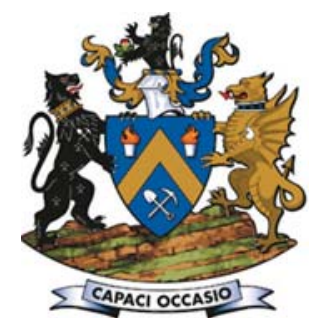

\title{
Methodology for assessing the benefits of grind control using particle size tracking technology for true on-line particle size measurement
}

\author{
by R. Maron*, C. O’Keefe*, and J. Sepulveda†
}

\section{Synopsis}

Despite the recognized influence of product fineness on line capacity and downstream process performance, few mineral concentrators today use particle size measurement of the final product in automatic control applications for particle size. 'Near-line' particle size instrumentation has existed for decades and is utilized in modern concentrators, but low availability and measurement frequency, due to problems with slurry sampling and transport systems, make these technologies unreliable for use in automatic control. An innovative technology for real-time particle size measurement on individual hydrocyclones, named CYCLONEtrac ${ }^{\mathrm{TM}}$ PST (particle size tracking), has been developed by CiDRA Minerals Processing, and proven in commercial installations, demonstrating near $100 \%$ availability with minimal maintenance requirements, thus overcoming the limitations of previous technologies. Potential stakeholders require convincing, reliable information regarding the economic value delivered by automatic grind control projects. In this paper we present a methodology for estimating such value with the CYCLONEtrac ${ }^{\mathrm{TM}}$ PST technology, and demonstrate unique opportunities to improve process stability and performance. Based on actual plant data accumulated over time, the evaluation approach assesses and highlights the significant potential process improvement that can be expected with the particle size tracking technology. As an extension of previous work, in this paper we present the key operating criteria for optimizing the production of valuable metals and increase mill throughput by identifying the optimal grind size. As this requires operating closer to the process boundaries, accurate real-time particle size measurements, such as those provided by the CYCLONEtrac ${ }^{\mathrm{TM}}$ PST system, become essential for achieving the maximum potential value from every operation. For further illustration, data from three different copper plants is compared.

Keywords

grind control, particle size, real-time measurement.

\section{Introduction}

Owners and operators of mineral concentrators constantly search for the most economically attractive way to increase the output of valuable metal. A logical approach is to identify a key parameter that has the greatest impact on the performance of multiple stages of the process and then use a holistic analysis methodology to determine how this key parameter can be controlled to optimize the final output.

Particle size is widely recognized as such a key parameter; however, very few concentrators today use real-time particle size measurements for automatic control of the final product size due, to a large extent, to the inherent limitations of existing technologies. 'Near-line' particle size measurement instrumentation, which has been used for decades, typically suffers from low availability because the fundamental measurement technologies were developed for other, less demanding industries and then adapted to the harsh environment of the typical mineral concentration process. An outline of the key differences among the available technologies used for particle size measurement in comminution circuits has been completed in previous work, and plant data has proven the benefits of measurements on individual cyclones - something that, until now, has not been possible (Cirulis, 2017).

The patented CYCLONEtrac ${ }^{\mathrm{TM}}$ PST particle size tracking (PST) technology was expressly designed for true on-line measurement of the product size on the overflow of individual hydrocyclones, almost continuously, avoiding the always cumbersome technique of taking representative slurry samples, thus overcoming several limitations of legacy technologies. Its simple acoustic-impact technology, rugged design, and absence of moving parts has solved the problem of low availability, thereby making real-time measurement and automatic control of ground product size a practical reality.

To assess the potential economic benefit of implementing this PST-based measurement and control system, the authors previously developed a methodology (Maron, O'Keefe, and Sepulveda, 2017) for determining the optimum final ground product size that results in the maximum net metal production (NMP),

* CiDRA Minerals Processing, Wallington, Connecticut, USA.

+ Consultants Ltd., Santiago, Chile.

(C) The Southern African Institute of Mining and Metallurgy, 2018. ISSN 2225-6253. This paper was first presented at the Copper Cobalt Africa, incorporating the 9th Southern African Base Metals Conference, 9-12 July 2018, Avani Victoria Falls Resort, Livingstone, Zambia. 


\section{Methodology for assessing the benefits of grind control using particle size tracking technology}
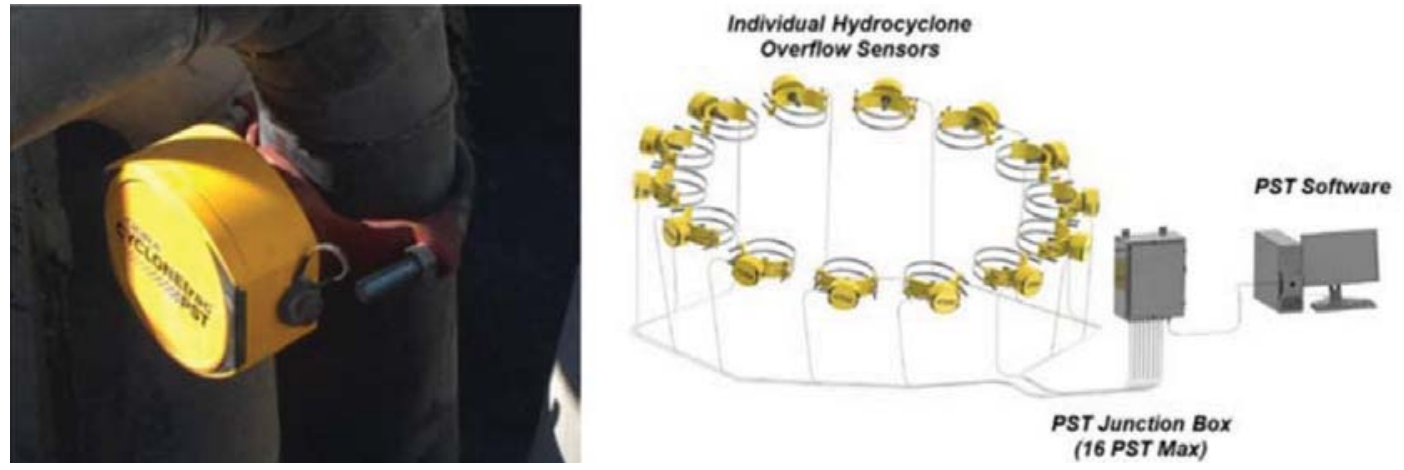

Figure 1-Particle size tracking mounted sensor head (left), and system with interconnections (right)

defined as the amount of valuable net metal produced per unit time. The method uses historical daily or hourly plant data for a minimum period of one year, ideally longer. Since that initial work, data-sets from two additional plants have been analysed. In all three cases, the analyses have shown that although there are significant potential gains to be realized from only reducing the variability in product size, there are additional and larger potential gains obtained from coarsening the product size, which enables the throughput of the plant to be increased, therefore proportionally increasing the NMP. However, this must be done in a stable and controlled manner to avoid downstream problems with material handling and to prevent roping conditions on individual hydrocyclones. This PST system provides the necessary information, not previously available, to more closely approach these process barriers in a safe operational way.

In this paper, we briefly review the technology, then examine three specific paths available to increase process performance, i.e., to maximize NMP, showing the key role of reliable ground product size measurements. We then briefly present the methodology used to determine the product size that maximizes NMP. Finally, we discuss three different realworld examples of applications of this methodology.

This approach to increasing NMP focuses on the secondary grinding or ball milling stage, for a good reason. In the 1980s and 1990s, the typical bottleneck in grinding circuits was the semi-autogenous (SAG) mill. However, significant improvements to SAG mills over the last two decades have now shifted the bottleneck to the ball mills. Thus, the ball mill classification circuit is the unit operation where the greatest potential NMP improvement can be obtained, with significant economic benefits.

\section{Impact-based real-time particle size measurement of cyclone overflow streams}

Acoustic impact-based PST is a unique method for measuring and controlling a reference product mesh size in cyclone overflow streams. The implementation of this technology is centred upon a sensor probe that is inserted into the overflow slurry stream via a two-inch $(50 \mathrm{~mm})$ hole in the overflow pipe. Particles in the slurry stream impact the surface of the probe, generating travelling stress waves within the probe. A sensor converts these travelling stress waves into an electrical signal, and proprietary signal processing techniques convert these signals into a particle size measurement that is output every four seconds. The sensor is constantly in contact with many particles in the slurry stream, thus obtaining information from orders-of-magnitude more particles than traditional sample-based technologies. Also, because of the location of the sensor downstream of the hydrocyclone and the presence of an air core at that point, the sensor produces no change in the back-pressure seen by the hydrocyclone and thus does not affect hydrocyclone performance. The probe has a useful life of approximately 18 months due to the abrasive wear caused by the direct slurry impact. The probe life is related to the particle hardness and size, which is obviously finer in the overflow stream than in the feed stream, thus enabling an acceptable probe life. Currently, the software provides a single reference mesh size to be incorporated into a process control strategy. Figure 1 shows the main components of the PST system.

\section{Improving process performance and the key role of product size}

The value analysis methodology, presented later, shows that to achieve the maximum increase in NMP, which is directly linked to net cash flow from the operation, we should increase throughput and coarsen the final product size, despite a possible decrease in recovery. The throughput equals the ratio of power to specific energy. The well-known Bond's Law relates the specific energy consumption used in size reduction to the feed and product sizes by a factor known as the Bond Work Index $(W i)$, which is a property of the ore (Bond, 1985). These two relationships can be combined, as shown in Equation [1], to show three parallel paths to increase throughput using the existing circuit, where $W$ is replaced by the 'operational work index' (Wio), which is the actual energy per ton as measured in the plant. Ideally, $W$ io should be less than $W$ i for an efficient grinding operation. A detailed explanation of these paths is well known and has been presented on various occasions, including by the current authors (Sepulveda, 2017), and is summarized in the following three subsections of this paper. 


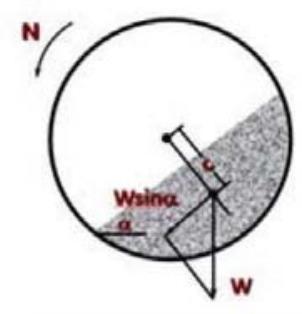

$P_{\text {net }}=\mathrm{c} \bullet \mathrm{W} \sin \alpha \cdot 2 \pi \mathrm{N}$

Torque

with:

$W=0.02832 \rho_{\text {ap }} J\left(\pi D^{2} / 4\right) L$

$c / D \cong 0.447-0.476 \mathrm{~J}$

$P_{\text {net }}=0.238 \mathrm{D}^{3.5}(\mathrm{~L} / \mathrm{D}) \mathrm{N}_{\mathrm{c}} \rho_{\mathrm{ap}}\left(\mathrm{J}-1.065 \mathrm{~J}^{2}\right) \sin \alpha$

Figure 2-Hogg and Furstenau model for mill power draw

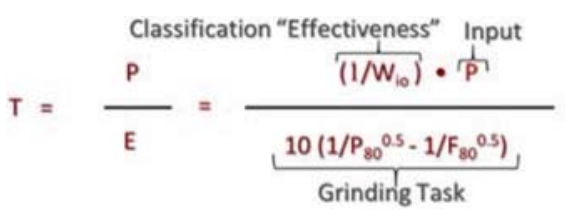

\section{Increase mill power draw}

The first path to increase throughput, as shown by Equation [1], is by increasing the power $(P)$ drawn by the ball mill. The well-known relationship shown in Figure 2 indicates how this can be accomplished. It follows that one should increase the mill charge level (J) and/or the mill speed (NC) whenever possible.

\section{Increase classification effectiveness}

The second path to increase throughput, as shown in Equation [1], involves the operational work index (Wio), which is affected by the response of the classifiers in their closed-circuit interaction with the ball mill. The Wio appears as a reciprocal in Equation [1], thus representing the 'effectiveness' of the classification, i.e., how the classifiers contribute to reducing the tons processed per unit of energy consumed. A more effective operation is that in which the Wio is reduced to its minimum possible value. This means that for optimal energy efficiency and throughput in an effective grinding process, the content of fine particles in the mill charge should be as low as possible for a given grinding task, thus reducing $W$ io. This may be achieved by operating the circuit under the following three conditions, which are sometimes referred to as the 'Fourth Law' criterion:
> Minimum \% solids overflow, only limited by the total water availability

> Maximum \% solids underflow, only limited by the undesirable 'roping' condition

> Maximum circulating load, only limited by the capacity of both the pump(s) and the mill itself to transport the required volume of slurry.

The collective goal of these three operating conditions is for the hydrocyclones to remove fine particles from the circuit as soon as they are reduced to the target size. In this way, the energy of the mill is directed to grinding the coarse particles that have not yet reached the final target grind size, rather than being used to overgrind particles that have already reached the final target size and should not be present in the mill.

\section{Relax grinding task}

The third and most effective way to increase throughput (T) is to relax the grinding task, which in Equation [1] is represented by the denominator that contains the feed size $\left(F_{80}\right)$ to the ball mill section and the product size $\left(P_{80}\right)$ discharged through the cyclone overflow. Equation [1] shows that bringing the $F_{80}$ and $P_{80}$ closer together, i.e., making the feed size smaller and/or making the product size coarser, will result in increased throughput (T). A graphical representation of the grinding task and the empirical relationship known as Bond's Law is shown in Figure 3 (left), where Wio represents the effective hardness of the ore. In the NMP optimization methodology, we focus on coarsening the $P_{80}$, which has a greater effect on increasing throughput than reducing the $F_{80}$ size, as shown in Figure 3 (right).

As the $P_{80}$ and throughput are increased, a reliable realtime measurement of product size, like the one provided by PST, becomes extremely valuable for implementing this method while avoiding problems as downstream process limits are approached. A typical process limit is when hydrocyclones enter a so-called roping condition as their underflow discharge capacity is exceeded. Figure 4 shows such an example of a ball mill classification circuit being pushed to a higher throughput and greater particle size - as measured by PST - until the point at which a hydrocyclone exceeds its operating limit and enters a roping condition. The
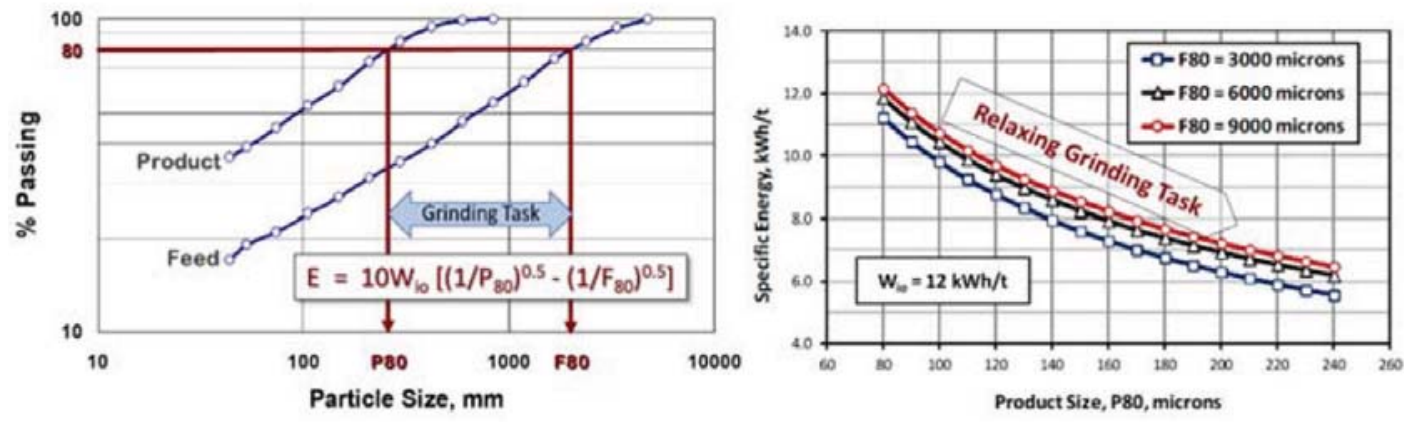

Figure 3-The 'grinding task' (left), and the effect of relaxing it (right) 


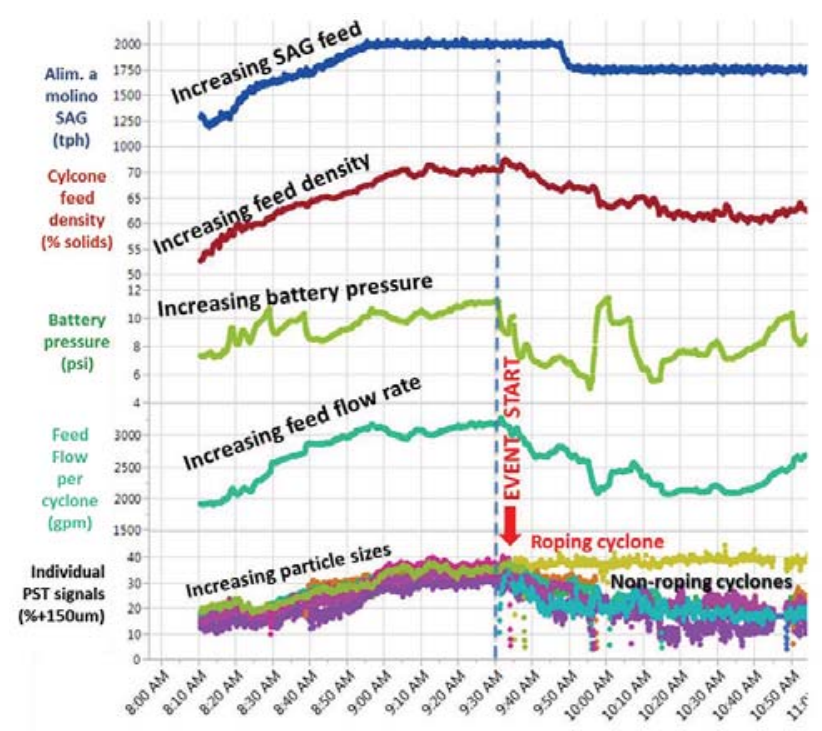

Figure 4-Hydrocyclone enters roping condition when process limit is exceeded

detrimental effect on the stability and performance of all the other cyclones in the battery is clearly seen, as the battery sends a large volume of coarse material to downstream flotation. The data was obtained after the installation of a real-time PST system but before it was used for control. The most relevant learning from this experience is that 'one wrong cyclone corrupts the whole battery of cyclones'.

\section{Value assessment methodology}

The objective of this methodology is to quantify the value of incorporating this on-line particle size measurement into the automatic process control system for a grinding/classification circuit using normally available historical plant data. The methodology uses daily data over a long enough time (ideally more than one year) to obtain a high-level assessment of the general plant operating performance, focusing mainly on throughput $(T)$ and recovery $(R)$, which are used to calculate the net production of valuable metal (NMP) - the primary generator of cash flow - giving the final objective function for process optimization purposes, as determined by the simple expression:

$\mathrm{NMP}=h T R$

where $h$ represents the head grade of valuable metal being recovered.

Inherent in this methodology is the usual observation that throughput $(T)$ and recovery $(R)$ are a strong function of product size, thus product size is chosen as the independent variable. Therefore, NMP is also a strong function of product size, thus there should exist an optimal product size that maximizes NMP and cash flow. Therefore, accurate measurement and control of product size is here highlighted as a necessity for effective process control. Correlations of throughput and recovery to other variables such as feed grade and ore hardness must also be examined in every case, whenever the proper information is available. Finally, operational plant constraints (like maximum tonnage or flow rates, product coarseness limitations for slurry transport, etc.) should also be taken into consideration as they may limit the maximum NMP values that can be consistently obtained.

\section{Throughput, recovery, and NMP vs. product size}

The minimum input data-set consists of the following four parameters, sampled at least once daily for a minimum of one year: mass per cent passing a target grind size $\left(e . g ., P_{80}\right.$ or percentage exceeding target mesh size), throughput per unit time, feed grade, and percentage net metal recovery. Once the
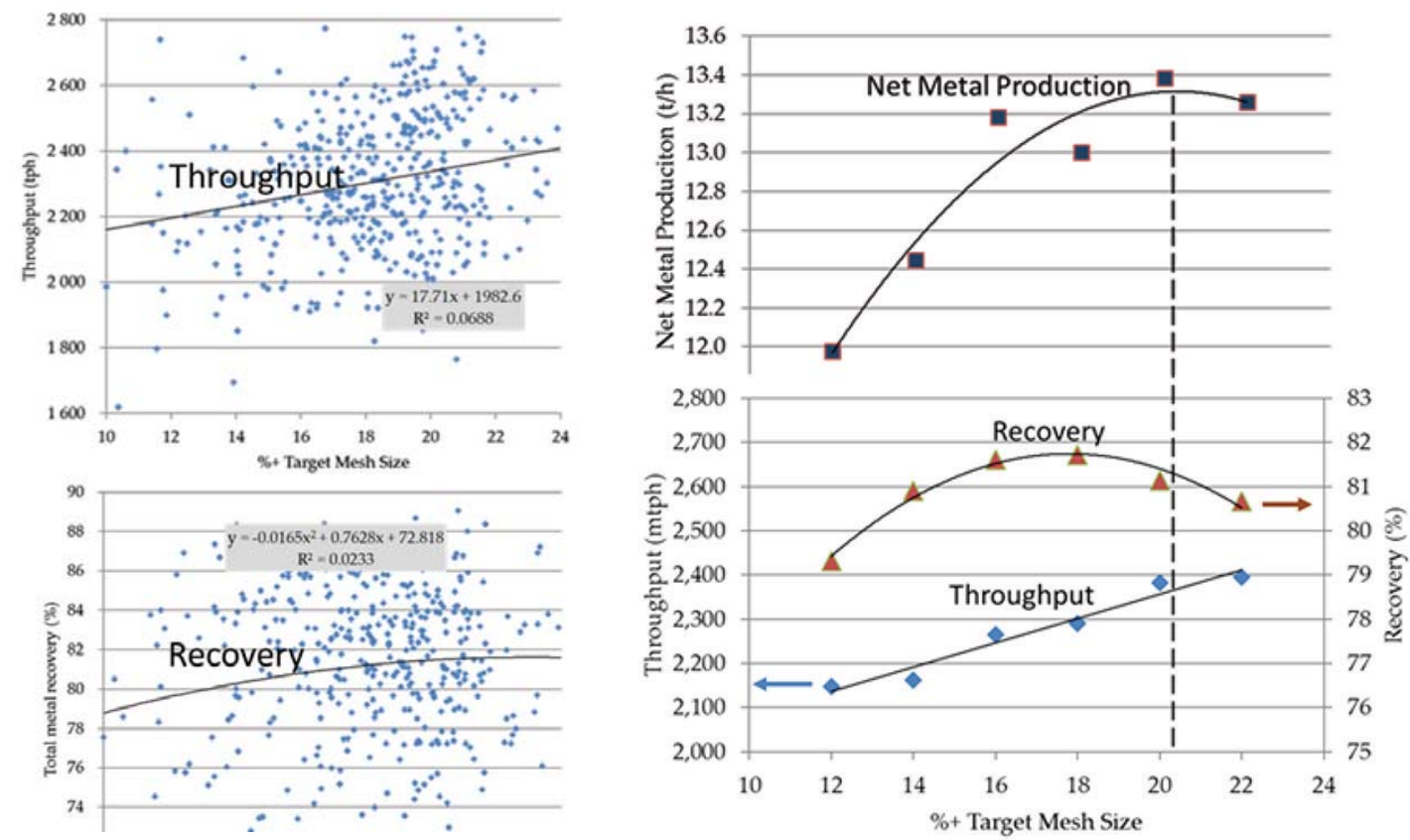

Figure 5-Throughput and recovery unfiltered (left), with data binning (right) with net metal production (NMP) objective function per Equation [1] 

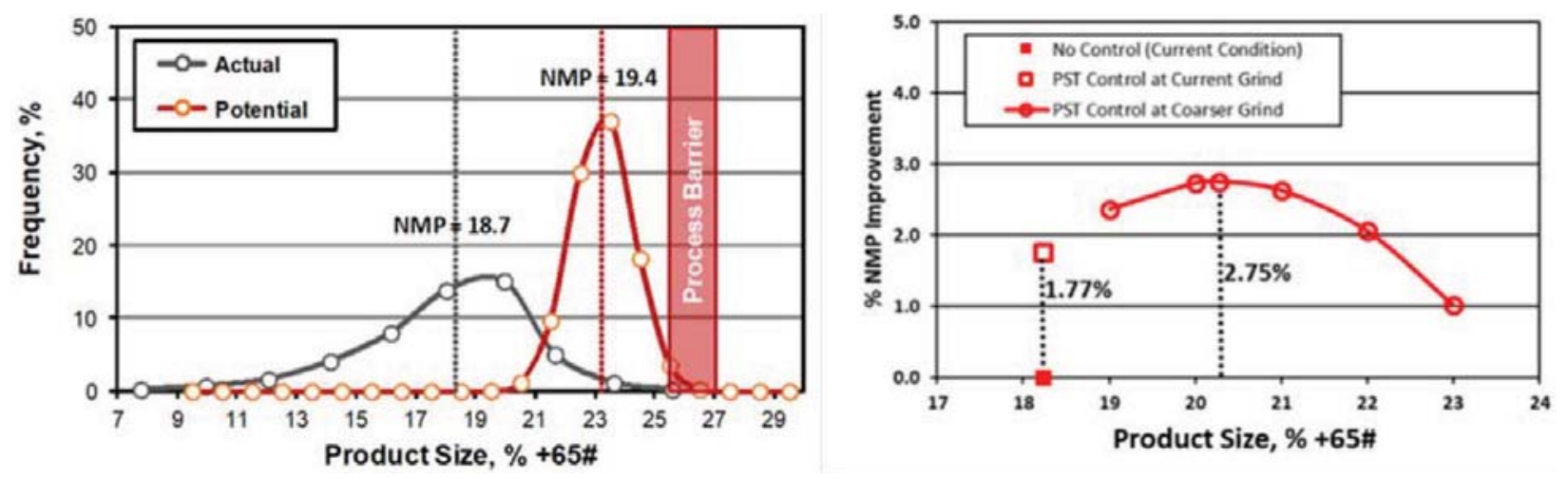

Figure 6-Actual and potential size distribution histograms and resultant NMPs (left) and associated potential percentage NMP improvement (right)

data-set is cleaned by filtering or data pre-processing, the throughput $(T)$ and recovery $(R)$ are plotted against target grind size, as shown in Figure 5 (left). These plots typically exhibit centrally weighted data clouds that make trend detection very unreliable. Both visual examination of such data clouds and regression fits are heavily influenced by a central grouping of data points, which are typically located around the plant operating points. To increase the influence of data points located further away from the normal operating points, data pre-processing can be used to reduce the number of points and allow for a clearer visual representation of the underlying trends. In our experience, this also increases the accuracy of the regression fits, particularly for low-order fit functions when the regression results are compared with other empirical data and firstprinciples models. The pre-processing used here, data binning, either through equal intervals or equal frequency, does allow for an increase in the influence of data outside the majority group of data points. Data binning partitions the horizontal axis and the data within these partitions is combined into a single number or category, which in this case was the mean of the data. In these particular data-sets, equal interval data binning was used, resulting in an increase in the influence of the data from the lower percentages of the target mesh size. The resulting equal interval means are shown in the throughput and recovery plots in Figure 5 (right).

The filtered throughput and recovery are then verified; i.e., throughput should show an increasing tendency with increasing particle size, and recovery should show a peak at a unique particle size, both as predicted by basic comminution and flotation practice. NMP can now be calculated, and its maximum identified as shown in Figure 5 (right).

In general, an important conclusion that can be drawn from the data, as shown in Figure 5, is that the maximum NMP is normally achieved by increasing throughput by coarsening the product size, usually at the expense of sacrificing some recovery. From this conclusion comes a challenge: how to measure grind size in a continuous, reliable way so that these measurements can be incorporated in the automatic control strategy, and thus prevent violation of the downstream process limitations imposed by particle size and/or material handling capabilities. Figure 6 (left) shows the actual particle size distribution without real-time particle size control, and the expected reduced variability and increased product size achievable by using real-time particle size control such as PST. Figure 6 (right) shows the potential incremental NMP improvements obtained by only reducing size variability at the current product size, and then coarsening the product size to a higher target size.
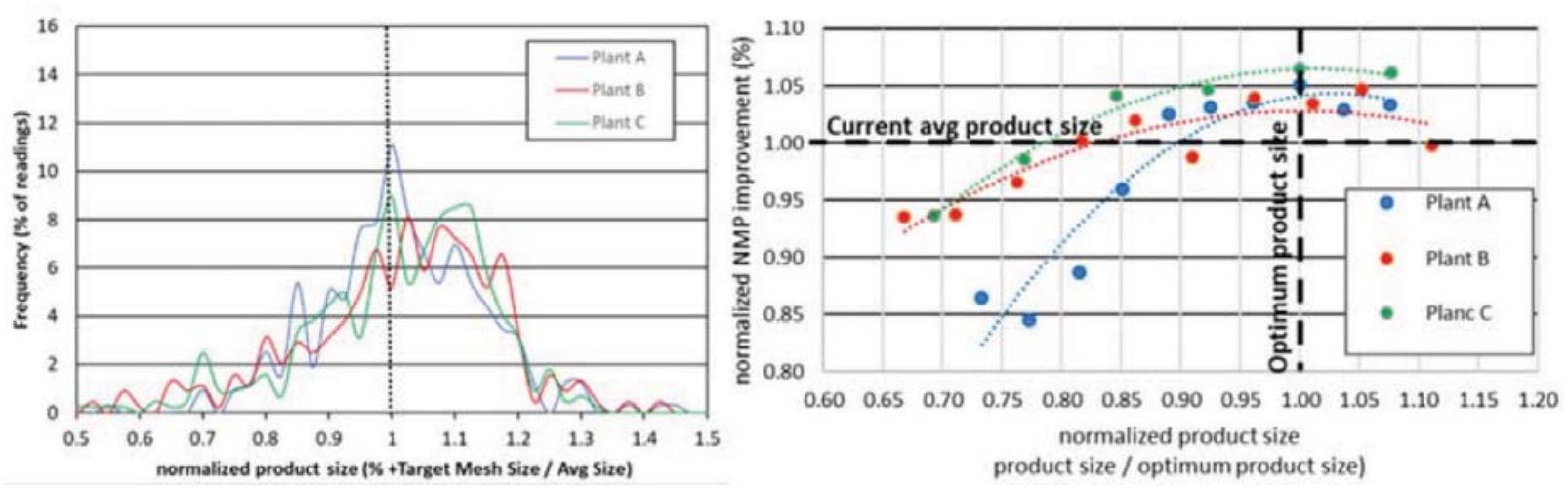

Figure 7-Normalized product size distribution without real-time size control (left); normalized NMP improvement (right) 


\section{Comparison of product size control in three plants}

In addition to the data-set analysed above, data-sets from two additional SAG and ball mill (SABC) circuits were analysed. To compare and benchmark the performance of plants without real-time product size control, normalized histograms of the product size distributions for the three plants are shown together in Figure 7 (left). For better comparison, the $x$-axis was normalized to the average product size and the $y$-axis normalized to the percentage of total number of readings. The data was plotted without filtering or data binning to reveal more of the data structure. It is interesting to observe that the natural process variability appears similar in all cases, considering that these are all large porphyry copper operations with similar grinding and classification circuits that do not include particle sized-based control strategies. It is to be expected that with particle size control strategies, the variability would be significantly reduced.

Figure 7 (right) shows the normalized NMP improvement for the plants, indicating that the current product size is normally in the range of $80-90 \%$ of the optimum size to achieve maximum NMP, and the expected NMP improvements are 3-6\% if the current average product size is increased to the optimum size.

\section{Conclusion}

Final ground particle size determines plant throughput and recovery, and thus the production of valuable metal that drives cash flow. The absence of a reliable real-time particle size measurement has for decades limited the implementation of automatic particle size control strategies that can enable mineral concentrator plants to maximize the production of valuable metal. The new, highly reliable realtime particle size measurement technology now enables plants to implement control strategies that permit them to grind coarser, increase throughput, and optimize metal production, thus more closely approaching process limits while monitoring and controlling product size to avoid downstream problems. We have presented the fundamental operational strategies that should be implemented to maximize valuable metal production, and a simple methodology to estimate the resulting economic benefits. Data from three plants showed a typical variation in product size in processes without automatic control based on realtime particle size measurement. The data also showed that significant increases in valuable metal production of several percentage points are possible. This methodology can be repeated on any mineral concentrator with a similar relationship between grind size, throughput, and recovery.

\section{References}

Bazin, C., Grant, R., Cooper, M., and Tessier, R. 1994. A method to predict metallurgical performances as a function of fineness of grind. Canadian Mineral Processors Annual Conference, CMP Proceedings 1964-1998. http://www.sciencedirect.com/science/article/pii/0892687594901155

Bond, F.C. 1985. Testing and calculations. SME Mineral Processing Handbook. Weiss, N.L. (ed.), Section 3A: General Aspects of Comminution. Society of Mining Engineers of the American Institute of Mining, Metallurgical, and Petroleum Engineers pp. 16-27.

Cirulis, D., Dunford, S., Snyder, J., Bartsch, E., Rothman, P., Maron, R. Newton, D., O'KeEfe, C., and Mercuri, J. 2015. Process optimization using real team tracking of coarse material in individual cyclone overflow streams. Proceedings of Metplant 2015 Conference, Perth, Western Australia, 7-8 September. Australasian Institute of Mining and Metallurgy, Melbourne.

CiRuLIs, D. 2017. Particle size tracking system vs. traditional measurement techniques. Engineering and Mining Journal, March. pp. 58-59.

Edwards, R., Vien, A., and Desbiens, A. (eds.). 1999. Application of a modelbased size-recovery methodology. Canadian Institute of Mining, Metallurgy and Petroleum, Montreal.

Maron, R., O'Keefe, C., and SepulvedA, J. 2017. Assessing the benefits of automatic grinding control using PST technology for true on-line particle size measurement. PROCEMIN-GEOMET 2017, Proceedings of the 13th International Mineral Processing Conference and 4th International Seminar on Geometallurgy. Gecamin, Santiago.

SEPulvedA, J.E. 2017. Operational guidelines for an energy efficient grinding/classification circuit. Pre-Seminar at PROCEMIN 2017, sponsored by the Coalition for Energy Efficient Comminution (CEEC), Santiago, Chile, 4 October 2017.

\section{The SAIMM Journal all you need to know! \\ Less $15 \%$ discount to agents only \\ 2. PRE-PAYMENT is required \\ is The Journal is printed monthly}

Surface mail postage included

मे ISSN 2225-6253

The SAIMM Journal gives you the edge!

* with cutting-edge research

* new knowledge on old subjects

* in-depth analysis

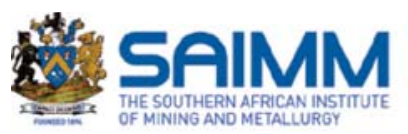

information please contact: Tshepiso Letsogo The Journal Subscription Department

Tel: 27-11-834-1273/7 •e-mail: saimmreception@saimm.co.za or journal@saimm.co.za Website: http://www.saimm.co.za

\section{A serious, 'must read' that equips you for your industry -Subscribe today!}

